\title{
Study of minimally invasive radiofrequency ablation of the ciliary body for the treatment of glaucoma in rabbits
}

\author{
BAOKE HOU* ${ }^{*}$ FENGXIANG WANG ${ }^{*}$, ZI YE, XIN JIN, YU FU and ZHAOHUI LI \\ Ophthalmology Department, Chinese PLA General Hospital, Beijing 100853, P.R. China
}

Received March 10, 2018; Accepted October 31, 2019

DOI: $10.3892 / \mathrm{mmr} .2019 .10906$

\begin{abstract}
The aim of the present study was to explore the safety and effectiveness of radiofrequency ablation (RFA) of the ciliary body for the treatment of glaucoma. A glaucoma model was established in New Zealand white rabbits, which were then treated with RFA of the ciliary body, utilizing an XL-1-type RF meter developed by the Chinese PLA General Hospital. After treatment, general ocular investigation, including ocular pressure was carried out, the anterior chamber was imaged via ultrasound biomicroscopy, and the pathological changes were observed via hematoxylin and eosin (H\&E) staining. It was determined that the glaucoma model was successfully established in the New Zealand white rabbit by inducing high intraocular pressure (IOP). After RFA treatment, ablation spots were observed but no clear anterior chamber reaction was found. The ablation group showed a steady and continuous decrease of IOP, which was significantly lower than the model group at days 3 and $7(\mathrm{P}<0.05)$. A sclera pathway was observed in the ablation site 1-day post-treatment, which had mostly recovered by day 7 . H\&E staining demonstrated shedding of the ciliary epithelium, and an unclear boundary between muscle layer and blood vessel at day 1 . This had fully recovered by day 14 , with clear ciliary layers and well-arranged muscle structures observed. The present study suggested that treatment with RFA could decrease IOP without substantial side effects in the glaucoma model in the rabbit. Therefore, it could be used as a strategy to control IOP and as a treatment for glaucoma in the clinic.
\end{abstract}

Correspondence to: Dr Zhaohui Li, Ophthalmology Department, Chinese PLA General Hospital, 28 Fuxing Road, Beijing 100853, P.R. China

E-mail: hbk_science@126.com

${ }^{*}$ Contributed equally

Key words: minimally invasive radiofrequency ablation, ciliary body, treatment, glaucoma

\section{Introduction}

Glaucoma is a progressive optic nerve disorder that is characterized by structural changes of the optic nerve head, and commonly accompanied by the development of visual functional defects (1). The World Health Organization has listed glaucoma as the second leading cause of irreversible blindness (2). According to World Health Organization statistics, $\sim 6,680$ million patients suffer from primary glaucoma and 600 million patients are affected by secondary glaucoma, resulting in 670 million patients exhibiting blindness (3). It is estimated by Quigley and Broman (4) that the number of patients with global primary glaucoma will grow to 7,960 million by the year 2020, while blindness caused by glaucoma will increase to 1,120 million.

It is widely known that elevated intraocular pressure (IOP) is the most critical risk factor that leads to optic nerve damage in glaucoma (5). Therefore, controlling IOP has been suggested as one of the main strategies to prevent the development of glaucoma from ocular hypertension, eliminate the progression of early glaucoma disease and block the occurrence of progressive visual function damage in the clinic (6-8). However, current clinically employed treatments for reducing IOP are not ideal. The treatment of glaucoma in clinical settings can be divided into drug therapy and surgical procedures (9). There are a wide range of drugs available that reduce IOP, but constant medication is required due to their limited effects. Consequently, a number of patients must resort to surgery after drug treatment (10). Anti-glaucoma surgery can be classified into two types: Procedures that facilitate the outflow of the aqueous humor, including aqueous humor shunts, and trabecular, goniotomy and iridotomy surgeries; and procedures that reduce aqueous humor secretion by destroying cells of the ciliary body, such as cyclocryotherapy and cyclophotocoagulation (11). However, the former group of procedures is flawed, due to scarring problems and their limited long-term effects, while the latter group lacks precise control and can only be utilized for patients with absolute glaucoma who have already lost their vision (12-15). Therefore, there is an urgent need to develop more safe and effective therapeutic approaches for glaucoma.

Radiofrequency ablation (RFA) is a new, minimally invasive technique (16). During treatment, microelectrodes are directly punctured into the focal site, and high-power radiofrequency energy is delivered inside through the catheter, making the 
local temperature high and resulting in irreversible coagulating necrosis (17). RFA has a number of advantages: It offers precise control, it is non-invasive and it demonstrates high effectiveness. Therefore, it has been applied in various departments, including cancer, cardiology, otorhinolaryngology, digestion, orthopedics, obstetrics, stomatology, surgery, physical therapy and dermatology (18). Currently, conductive keratoplasty has also been utilized in the clinic for the treatment of presbyopia, in which the radiofrequency energy is employed to change the shape of the eye's surface $(19,20)$. The successful application of conductive keratoplasty indicates that radiofrequency treatment may be safe and effective for ocular diseases.

In the present study, the minimally invasive technique of radiofrequency catheter ablation was used, guided by ultrasound biomicroscopy (UBM), to treat glaucoma. The ciliary body was specifically targeted and ablated, following which the production of the aqueous humor was reduced, which significantly lowered IOP. RFA technology not only controls IOP precisely, reliably, safely and durably, but it is also simple and repeatable, with a relatively low cost (21). Thus, it was hypothesized that minimally invasive RFA may be a highly desirable treatment procedure for glaucoma, from a social and economic standpoint.

\section{Materials and methods}

Experimental animals. A total of $30 \mathrm{New}$ Zealand rabbits (age, 4-6 months; weight, 2.0-2.5 kg; sex, 16 female and 14 male) were purchased from the Animal Research Center of the Chinese PLA General Hospital. Eye disease was excluded before research. All rabbits were allowed free access to food and water, and lived in an environment with 12-h light/dark cycles, a temperature of $25^{\circ} \mathrm{C}$ and $\sim 50 \%$ humidity. Animals were randomly divided into 3 groups: The model group $(n=10)$, in which the drug was injected into the anterior chamber to induce glaucoma, but no treatment was applied; the RFA group $(n=10)$, where rabbits were treated with RFA after the induction of glaucoma; and the sham group $(n=10)$, in which saline instead of the drug was injected into the anterior chamber. All protocols were conducted in accordance with the National Institutes of Health Guidelines for the Care and Use of Laboratory Animals (22) and approved by the Institutional Animal Care and Use Committee of the Chinese PLA General Hospital.

Establishment of the glaucoma model in rabbits. Glaucoma was induced by injection of carbomer and dexamethasone solution. Rabbits were anesthetized after intravenous administration of sumianxin $(0.1 \mathrm{ml} / \mathrm{kg}$; consists of dihydroetorphine hydrochloride, dimethylaniline thiazole, ethylenediam-inetetraacetic acid and haloperidol) (23) and topical application of $0.5 \%$ dicaine (24). Firstly, the corneal limbus of the paranasal side was punctured with a 1-ml syringe needle to form a pinhole. Then, $1 \mathrm{ml}$ of aqueous humor was extracted from the corneal limbus on the temporal side, followed by an injection of $0.2 \mathrm{ml}$ of carbomer solution containing $0.3 \%$ carbomer and $0.025 \%$ dexamethasone (Sigma-Aldrich; Merck KGaA). After $24 \mathrm{~h}$, IOP was checked and a value of IOP $>22 \mathrm{mmHg}$ was used to determine the successful establishment of the glaucoma model (success ratio was $85 \%$ in the present study).
RFA treatment for glaucoma. RFA surgery was performed based on an XL-1-type RF exposure system developed by the Chinese PLA General Hospital (with independent intellectual property rights), which can produce $\sim 300-500-\mu \mathrm{m}$ wide, $1-1.5-\mathrm{mm}$ deep tissue ablation. After local anesthesia ( $0.5 \%$ dicaine, one drop) (24) combined with general anesthesia (sumianxin mixture, $0.1 \mathrm{ml} / \mathrm{kg}$ ) (23), 5 model eyes were randomly selected, and the sclera was punctured at $1.5 \mathrm{~mm}$ from the corneal margin with a depth of $1 \mathrm{~mm}$. This was exposed to a probe that generated a $500-\mathrm{KHz}$ radiofrequency field at specific absorption rate of $0.5-0.8 \mathrm{~W} / \mathrm{kg}$. Output power was set at 0.6-1.0 W, duration was 1-2 sec and the number of ablation sites was 8 .

IOP measurement. Under local anesthesia, IOP was measured before and on days 1, 3 and 7 post-RFA with a Schiotz tonometer (Rudolf Riester GmbH). Scalp acupuncture connected to the Schiotz tonometer was inserted into the anterior chamber through the margin between the sclera and the cornea. After 5 min of stabilization, the value was read three times and the average value was calculated. The value obtained before ablation surgery was considered as the basal level of IOP.

Investigation of changes to the eyeball and anterior segments with UBM and slit lamp. Investigation with the slit lamp was carried out before surgery and on days 1, 3 and 7 post-surgery. Before investigation, rabbits were anesthetized with $3.5 \%$ sodium phenobarbital (35 mg/kg) (25). After the corneal reflex and corneal touch reflection disappeared, the transparency of the cornea, shades of the anterior chamber, the oscillation or opacity of the aqueous humor, and damages to the iris and lens were checked. Subsequently, our self-designed lens-water bath transformation system (patent no. ZL201420207350.0) was utilized to investigate the rabbit eyes with UBM for the purpose of assessing the influence of ablation on the ciliary body. During the UBM test, $0.2 \mathrm{ml} \mathrm{0.1 \%} \mathrm{(w/v)} \mathrm{hyaluronic} \mathrm{acid}$ sodium drops were administered to the junctival sac with the external optic cup utilized.

Histopathological assay after RFA. On 1, 3, 7 and 14 days after RFA, 2 rabbits on each day were anesthetized with an intravenous injection of $3.5 \%$ sodium phenobarbital $(35 \mathrm{mg} / \mathrm{kg})$ and sacrificed by ear vein air embolism (10-20 ml air). Following this, the eyeball was removed and $0.1 \mathrm{ml} 4 \%$ formaldehyde was injected into the vitreous body through the site of the optic nerve. The eye was then soaked in $4 \%$ formaldehyde at $25^{\circ} \mathrm{C}$ for $24 \mathrm{~h}$. The cornea, lens and posterior segment were removed from the fixed eyes. Then, the ciliary body and the sclera were collected, embedded in paraffin and cut into $5-\mu \mathrm{m}$ sections. They were stained with hematoxylin for $5 \mathrm{~min}$ and eosin for 3 min, respectively, to observe the pathological changes in the retinal ganglion cells using light microscopy (magnification, $\mathrm{x} 400)$.

Statistical analysis. SPSS 17.0 software (SPSS, Inc.) was used for data analysis. Results of IOP were expressed as mean \pm SD. Comparisons of IOP among three groups were performed using one-way ANOVA, followed by LSD post hoc test for multiple comparisons. $\mathrm{P}<0.05$ was considered to be statistically significant. 


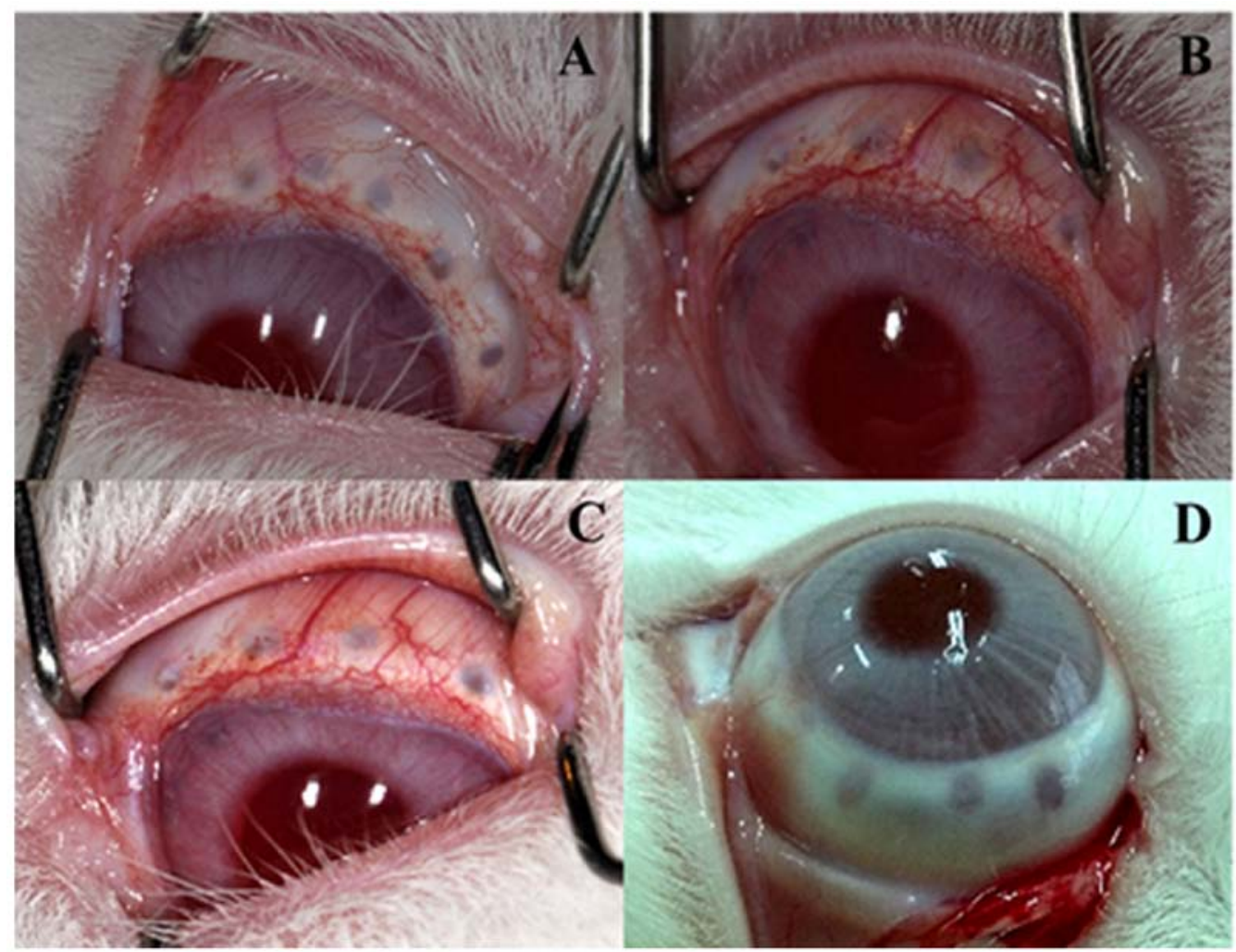

Figure 1. Melting spot investigation of the ciliary body in glaucoma model rabbits. Melting spots were observed at (A) 1 , (B) 3 , (C) 7 and (D) 14 days after radiofrequency ablation.

\section{Results}

General observation after RFA. After radiofrequency catheter ablation of the ciliary body, the eyes were examined with UBM and a slit lamp. A melting spot was observed (Fig. 1), but no puncture point penetrating the ciliary body into the eye was shown in the UBM check. Slit-lamp examination results showed no significant anterior chamber reaction.

Changes in IOP after RFA. In comparison with the sham group, rabbits of the glaucoma model showed $\sim 2.5 \mathrm{x}$ higher IOP $(\sim 33 \mathrm{mmHg})$. At 1 day after RFA of the ciliary body, IOP was still higher than in the sham group $(\sim 15 \%)$, but 3 days post-RFA, IOP was decreased by $\sim 40 \%$, and significantly lower than the model group $(\mathrm{P}=0.047)$. At day 7 , IOP of the RFA group continued to decrease $(\sim 25 \%)$, although it was not completely restored to the level of the sham group. However, it was significantly lower compared with the model group $\left(\mathrm{P}=3.64 \times 10^{-4}\right.$; Fig. 2$)$.

UBM investigation of anterior segment after RFA. Stable UBM images were obtained by utilizing the objective-bath conversion device coupled with UBM that we developed. Based on this method, the changes of the anterior segments at different time points after ablation were investigated. Fig. 3A shows the anterior segments of the sham group, in which the corneal, anterior chamber angle, iris and the ciliary body could be seen clearly. Fig. 3B-D are days 1, 3 and 7 post-ablation, respectively. At 1 day post-RFA, a straight RFA pathway was displayed in the ciliary body, damages within the ciliary

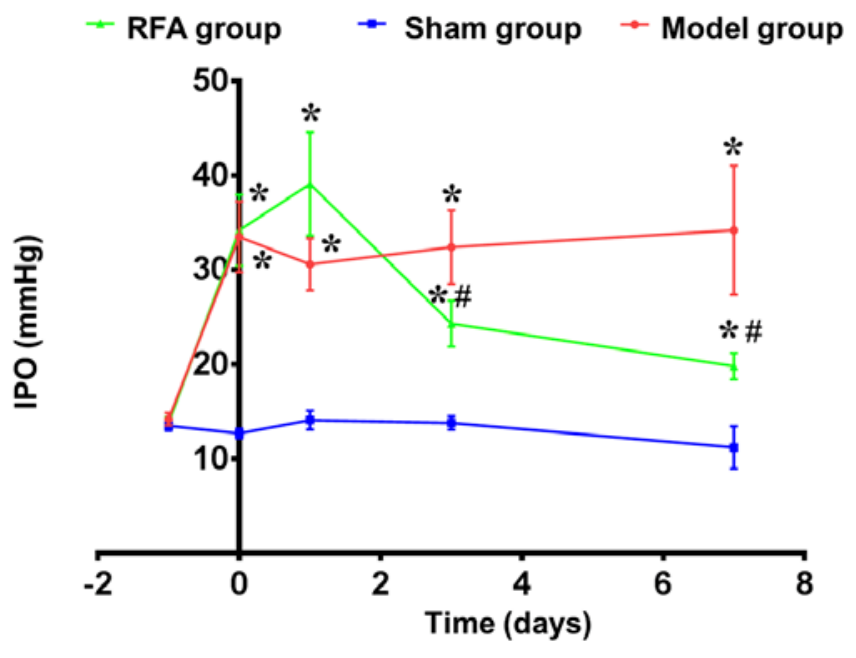

Figure 2. Changes in IOP in the RFA, sham and model groups at 1, 3 and 7 days post-RFA of the ciliary body in glaucoma model rabbits. ${ }^{*} \mathrm{P}<0.05$ vs. sham group; ${ }^{\text {"}} \mathrm{P}<0.05$ vs. model group. IOP, intraocular pressure; RFA, radiofrequency ablation.

body can be seen, but no penetration of the ciliary body was observed (Fig. 3B). At day 3, ablation-caused damage had decreased, peripheral damage was repaired and the ablation pathway showed a rounded rectangular shape (Fig. 3C). At day 7 post-RFA, the area of damages continued to shrink, displaying an oval shape (Fig. 3D).

$H \& E$ staining and pathological changes after RFA. At 1, 3, 7 and 14 days post-RFA of the ciliary body, pathological changes 

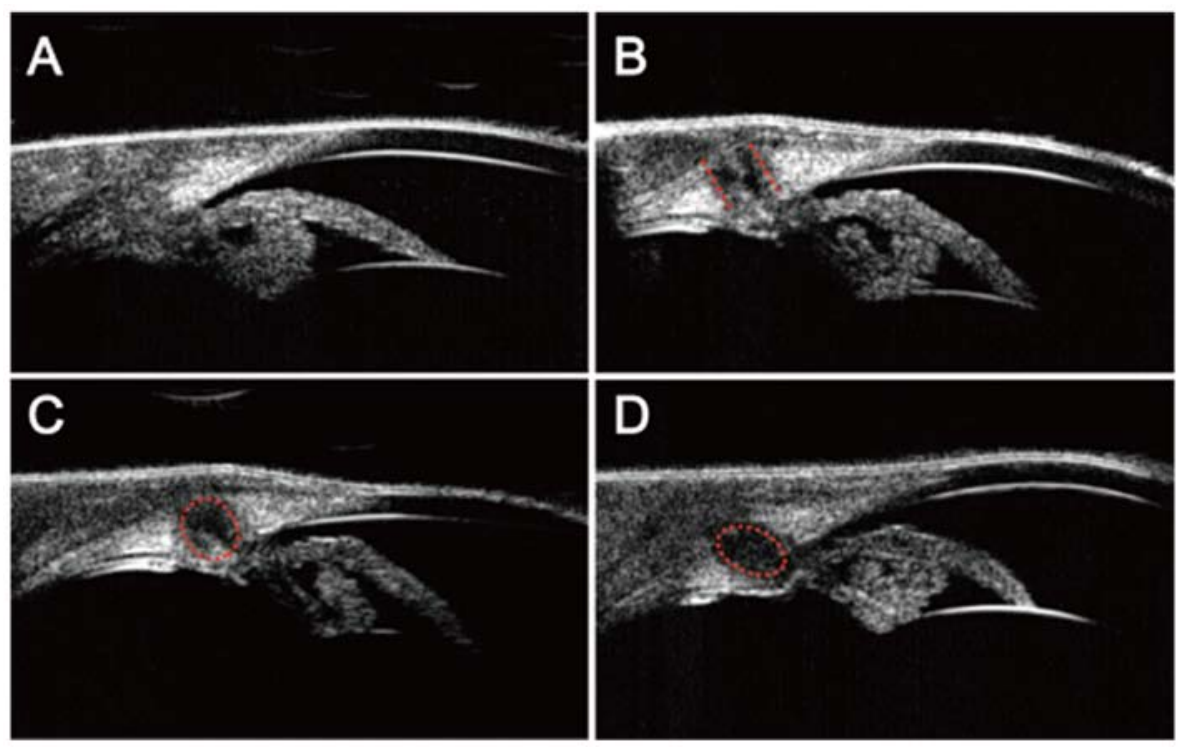

Figure 3. Ultrasound biomicroscopy investigation of the anterior segment after RFA. (A) Anterior segment of the sham group. (B) 1 , (C) 3 and (D) 7 days post-RFA. RFA, radiofrequency ablation. Red dotted lines signify the channel of radiofrequency ablation probe into ciliary body at different time points.

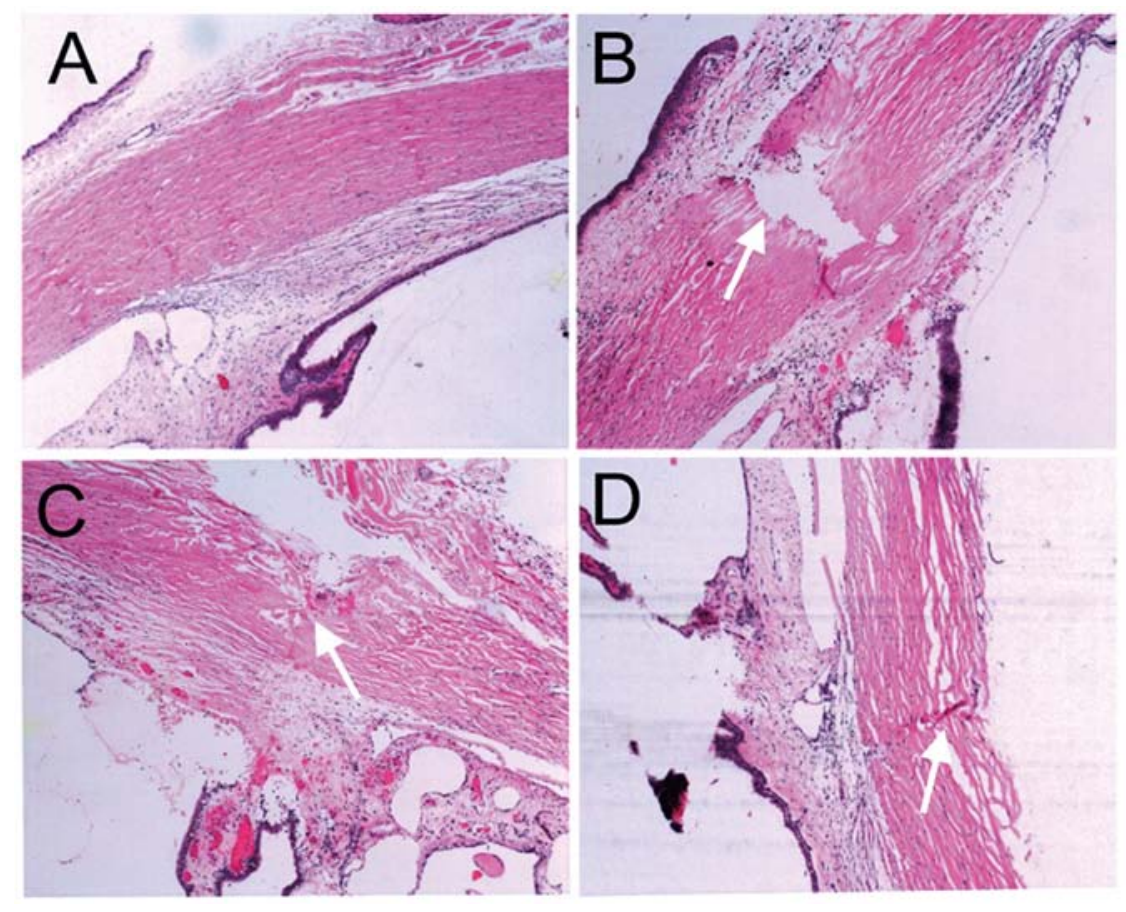

Figure 4. Hematoxylin and eosin staining and pathological changes after RFA of the ciliary body in rabbits. Images shown at (A) 1, (B) 3 , (C) 7 and (D) 14 days post-RFA. RFA, radiofrequency ablation. The white arrows show the channel of radiofrequency ablation probe into ciliary body at different time points. Magnification, $\mathrm{x} 100$.

were assessed in H\&E stained slices with a light microscope. The sham group displayed a normal chamber angle, the muscle layers of the ciliary body were arranged densely and regularly, and the epithelium was intact (Fig. 4A). At day 1 post-RFA, the sclera fiber was fractured at the site of the ablation pathway and the endothelium of the ciliary body had become disordered, but the ciliary body was not penetrated (Fig. 4B). At 7 days post-RFA, the layer boundaries of the ciliary body had become clear, muscle layer fibers on the ablation pathway were arranged loosely and the scar had healed (Fig. 4C). At 14 days post-RFA, the scleral ablation pathway had almost healed, with only a few cells infiltrated, the ciliary body had healed and the epithelium was integrated (Fig. 4D).

\section{Discussion}

RFA was first introduced by Harvey Cushing and WT Bovie for the clinical treatment of neurological diseases, followed by Huang in 1987 (26) who applied it in the treatment of arrhythmia disease (27). In the 21 st century, RFA technologies began to be widely utilized in minimally invasive surgery for the treatment of various diseases (18). Based on the characteristics of glaucoma 


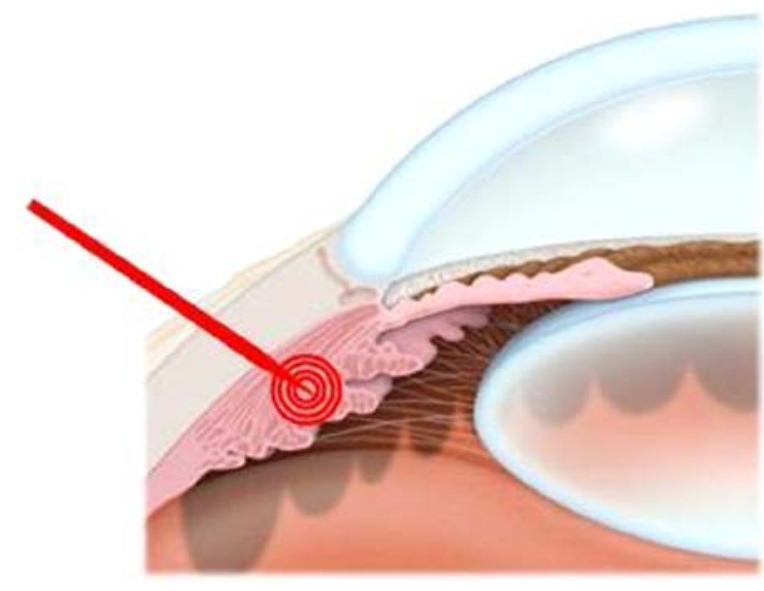

Figure 5. Proposed radiofrequency ablation of the ciliary body using our self-designed ciliary ablation handle.

and the structure of eyes, the aim of the present study was to find out whether RFA technology could also be applicable in the treatment of glaucoma, which has not been reported previously. In the current study, a New Zealand white rabbit model of glaucoma was used to examine the potential of RFA of the ciliary body for the treatment of glaucoma. As expected, the results of the present study showed that region-specific ablation of the ciliary body could reduce the production of aqueous humor, which is the cause of increased ocular pressure, and gradually restore IOP to a normal level over a long period of time.

Radiofrequency (RF) techniques have been used to heat biological tissues for a number of years. Laser ablation (LA) is also a thermal ablation technique (28). However, their heating mechanisms are different. In RFA, resistive heating is generated by the agitation of ions, due to the alternating directions of the electric field, while in LA, hyperthermia is produced by increased light absorption and heat conduction (29). To achieve a reduction in IOP, the output power required is $1.5-2 \mathrm{~W}$ for 1.5-2 sec, and the temperature of the tissue is set between $70^{\circ} \mathrm{C}$ and $110^{\circ} \mathrm{C}$ in LA (30), which seem to be higher than those used for RFA in the present study (output power, $0.6 \mathrm{~W}$; duration, $0.6 \mathrm{sec}$; temperature, $55-75^{\circ} \mathrm{C}$ ). Thus, LA surgery could induce an inflammatory reaction and lead to several related complications, such as cystoid macular edema, hyphema and persistent hypotony (31). In addition, RFA is completed by moving the electrode under the guide of UBM and thereby precisely acting on the target lesion regions (32), and thermometers can be incorporated into the tips of the electrodes that allow for the continuous monitoring of tissue temperatures (33). These both prevent underlying damage to the adjacent structures. Furthermore, the fiber used in the LA may be difficult to place into the target sites, so the treatment efficiency and safety may be lower than RFA. Therefore, RFA may be more suitable for the treatment of glaucoma.

The technique of minimally invasive RFA demonstrated here has a high potential for application in the clinical treatment of glaucoma $(34,35)$. First of all, the rabbit's eye has the power to proliferate and self-repair (36), while its size is comparable to the human eye. The body size of the rabbit is small, which facilitates the use of the slit lamp microscope. These advantages make the rabbit model of glaucoma more suited to study common clinical issues than other animal models, and consequently it is widely used for the preclinical study of glaucoma (37). This is the reason that the ocular hypertension model in rabbits was chosen for the present study. Secondly, invasive RFA technology can not only control IOP precisely, reliably, safely and durably, but it is also easy to operate, it is a repeatable therapy, and it is relatively less expensive, resulting in a high socio-economic value.

Since standard wands were used, some scleral damage occurred after ablation. This damage could be seen in the images obtained using ultrasound biomicroscopy, UBM anterior segment imaging and pathological imaging. To this end, a new type of RF wand was designed for ablation of the ciliary body (patent pending). This new wand is loaded with a specially designed insulating sheath that can more effectively protect the tissue around the sclera puncture track, making ablation more restricted to the needle tip of the wand or handle and thus, the ciliary processes (Fig. 5).

However, there are some limitations to the present study. Firstly, this was only an animal model study and further clinical studies with short or long-term follow up are also needed to confirm the effectiveness and safety of RFA. Risks may include cataract development, cognitive effects and possible carcinogenic effects due to the frequency used, which is within the range of $30 \mathrm{kHz}$ to $300 \mathrm{GHz}$. Secondly, a direct comparison between RFA and LA for glaucoma is lacking.

The results of the present study indicated that XL-1 meter-based RFA of the ciliary body for controlling ocular pressure in patients with glaucoma is mostly safe and effective, showing only a few anterior chamber reactions and almost no influence on intraocular structures. In further studies, the new handle for the ciliary body ablation, the dose-effect relationship of the ciliary body ablation in the treatment of glaucoma and the application of RFA of the ciliary body for treatment of absolute glaucoma in clinical settings will be investigated.

\section{Acknowledgements}

The authors would like to thank Dr Xinglin Wang (Chinese PLA General Hospital) for assistance using the RF instruments and Dr Zhigang Song for helping to analyze the pathological results (Chinese PLA General Hospital).

\section{Funding}

The present study was supported by The National Key Research and Development Project (grant no. 2016YFC1305504).

\section{Availability of data and materials}

The datasets used and/or analyzed during the current study are available from the corresponding author on reasonable request.

\section{Authors' contributions}

ZL conceived and designed the experiments. ZY analyzed the data. $\mathrm{BH}$ and $\mathrm{FW}$ performed the experiments and wrote the article for publication. ZY, XJ, YF and ZL helped to perform the experiments. All authors read and approved the final version of the manuscript to be published. 


\section{Ethics approval and consent to participate}

All protocols were conducted in accordance with the National Institutes of Health Guidelines for the Care and Use of Laboratory Animals and approved by the Institutional Animal Care and Use Committee of Chinese PLA General Hospital.

\section{Patient consent for publication}

Not applicable.

\section{Competing interests}

The authors declare that they have no competing interests.

\section{References}

1. Haffner DS, Smedley GT, Tu H and Burns TW: Devices and methods for glaucoma treatment: US Patent 9,597,230. Filed March 20, 2017; issued February 8, 2018.

2. Gupta N and Yücel YH: Glaucoma as a neurodegenerative disease. Curr Opin Ophthalmol 18: 110-114, 2007.

3. Cook C and Foster P: Epidemiology of glaucoma: What's new? Can J Ophthalmol 47: 223-226, 2012.

4. Quigley HA and Broman AT: The number of people with glaucoma worldwide in 2010 and 2020. Br J Ophthalmol 90: 262-267, 2006.

5. Pang JJ, Frankfort BJ, Gross RL and Wu SM: Elevated intraocular pressure decreases response sensitivity of inner retinal neurons in experimental glaucoma mice. Proc Natl Acad Sci USA 112: 2593-2598, 2015.

6. Roy Chowdhury U and Fautsch MP: Intracranial pressure and its relationship to glaucoma: Current understanding and future directions. Med Hypothesis Discov Innov Ophthalmol 4: 71, 2015.

7. Stevens A, Iliev ME, de Jong L, Grobeiu I and Hommer A: A combined analysis of four observational studies evaluating the intraocular pressure-lowering ability and tolerability of bimatoprost $0.01 \%$ in patients with primary open-angle glaucoma or ocular hypertension. Clin Ophthalmol 10: 635-641, 2016.

8. Heijl A, Leske MC, Bengtsson B, Hyman L, Bengtsson B and Hussein M; Early Manifest Glaucoma Trial Group: Reduction of intraocular pressure and glaucoma progression: Results from the Early Manifest Glaucoma Trial. Arch Ophthalmol 120: 1268-1279, 2002.

9. Dietlein TS, Hermann MM and Jordan JF: The medical and surgical treatment of glaucoma. Dtsch Arzteblatt Int 106: 597-605, 2009.

10. Calissendorff BM: Costs of medical and surgical treatment of glaucoma. Acta Ophthalmol Scand 79: 286-288, 2001.

11. Rosenfeld C, Price MO, Lai X, Witzmann FA and Price FW Jr: Distinctive and pervasive alterations in aqueous humor protein composition following different types of glaucoma surgery. Mol Vis 21: 911-918, 2015.

12. Stryker JE, Beck AD, Primo SA, Echt KV, Bundy L, Pretorius GC and Glanz K: An exploratory study of factors influencing glaucoma treatment adherence. J Glaucoma 19: 66-72, 2010.

13. Baudouin C, Denoyer A and Rosténe W: Glaucoma today: Detection and therapeutic progress. Biol Aujourdhui 207: 87-95, 2013 (In French).

14. Ahmed S, Khan Z, Si F, Mao A, Pan I, Yazdi F, Tsertsvadze A, Hutnik C, Moher D, Tingey D, et al: Summary of glaucoma diagnostic testing accuracy: An evidence-based meta-analysis. J Clin Med Res 8: 641-649, 2016.

15. Gedde SJ and Vinod K: Resident surgical training in glaucoma. Curr Opin Ophthalmol 27: 151-157, 2016.

16. Wahl DR, Stenmark MH, Tao Y, Pollom EL, Caoili EM, Lawrence TS, Schipper MJ and Feng M: Outcomes after stereotactic body radiotherapy or radiofrequency ablation for hepatocellular carcinoma. J Clin Oncol 34: 452-459, 2016.

17. Haemmerich D: Biophysics of radiofrequency ablation. Crit Rev Biomed Eng 38: 53-63, 2010.

18. Goldberg SN: Radiofrequency tumor ablation: Principles and techniques. Multi-treatment modalities of liver tumours. Springer, Boston, MA: 87-118, 2002.
19. Ye PP, Xu W, Xu HS, Li ZC, Shi JT, He FY and Yao K: Conductive keratoplasty: An approach for the correction of residual hyperopia in post-lasik pseudophakia. Int J Ophthalmol 5: 630-633, 2012.

20. Sy ME, Kovoor TA, Tannan A, Choi D, Deng SX, Danesh J and Hamilton DR: Combined astigmatic keratotomy and conductive keratoplasty to correct high corneal astigmatism. J Cataract Refract Surg 41: 1050-1056, 2015.

21. Cheng $\mathrm{CH}$, Sanders GD, Hlatky MA, Heidenreich P, McDonald KM, Lee BK, Larson MS and Owens DK: Cost-effectiveness of radiofrequency ablation for supraventricular tachycardia. Ann Intern Med 133: 864-876, 2000.

22. Institute of Laboratory Animal Resources (US). Committee on Care, the 2. Laboratory Animal Husbandry in Use of Laboratory Animals. Guide for the care and use of laboratory animals. US Department of Health and Human Services, Public Health Service, National Institutes of Health, 1986.

23. Yan D, Zhang J, Liang W, Sun J, Liu BY, Tian W and Cheng XG: Magnetic resonance imaging and histopathological analysis of experimental muscle injuries in a rabbit. Biomed Environ Sci 26: 841-848, 2013.

24. Zhang ZY, Chu RY, Zhou XT, Dai JH, Sun XH, Hoffman MR and Zhang XR: Morphologic and histopathologic changes in the rabbit cornea produced by femtosecond laser-assisted multilayer intrastromal ablation. Invest Ophthalmol Visual Sci 50: 2147-2153, 2009.

25. Balazs T, Hooper W, Farber TM, Van Loon EJ, Earl FL and Weinberger MA: Studies on the effects of anticonvulsant drugs on the activity of vitamin D in rats and dogs. Toxicol Appl Pharmacol 29: 47-52, 1974.

26. Huang SK, Bharati S, Graham AR, Lev M, Marcus FI and Odell RC: Closed chest catheter desiccation of the atrioventricular junction using radiofrequency energy-a new method of catheter ablation. J Am Coll Cardiol 9: 349-358, 1987.

27. Miao Y, Ni Y, Yu J, Zhang H and Marchal G: Evaluation of radiofrequency ablation as an alternative for the treatment of brain tumor in rabbits. J Neurooncol 56: 119-126, 2002.

28. Cohen A, Wong SH, Patel S and Tsai JC: Endoscopic cyclophotocoagulation for the treatment of glaucoma. Surv Ophthalmol 62: 357-365, 2017.

29. Di Costanzo GG, Tortora R, D'Adamo G, De Luca M, Lampasi F, Addario L, Galeota Lanza A, Picciotto FP, Tartaglione MT, Cordone G, et al: Radiofrequency ablation versus laser ablation for the treatment of small hepatocellular carcinoma in cirrhosis: A randomized trial. J Gastroenterol Hepatol 30: 559-565, 2015.

30. Fenelon G, Pereira KP and de Paola AA: Epicardial radiofrequency ablation of ventricular myocardium: Factors affecting lesion formation and damage to adjacent structures. J Interve Card Electrophysiol 15: 57-63, 2006.

31. Kaplowitz K, Kuei A, Klenofsky B, Abazari A and Honkanen R: The use of endoscopic cyclophotocoagulation for moderate to advanced glaucoma. Acta Ophthalmol 93: 395-401, 2015.

32. Vogl TJ, Farshid P, Naguib NN, Darvishi A, Bazrafshan B, Mbalisike E, Burkhard T and Zangos S: Thermal ablation of liver metastases from colorectal cancer: Radiofrequency, microwave and laser ablation therapies. Radiol Med 119: 451-461, 2014.

33. Ha EJ, Baek JH, Kim KW, Pyo J, Lee JH, Baek SH, Døssing H and Hegedüs L: Comparative efficacy of radiofrequency and laser ablation for the treatment of benign thyroid nodules: Systematic review including traditional pooling and bayesian network meta-analysis. J Clin Endocrinol Metab 100: 1903-1911, 2015.

34. Bong JJ, Kumar R and Spalding D: A novel technique of partial splenectomy using radiofrequency ablation. J Gastrointest Surg 15: 371-372, 2011.

35. Nierkens S, den Brok MH, Ruers TJ and Adem GJ: Radiofrequency ablation in cancer therapy: Tuning in to in situ tumor vaccines. Tumor Ablation. Springer, Dordrecht, 39-59, 2013.

36. Abrams GW, Topping TM and Machemer R: Vitrectomy for injury: The effect on intraocular proliferation following perforation of the posterior segment of the rabbit eye. Arch Ophthalmol 97: 743-748, 1979.

37. Esson DW, Neelakantan A, Iyer SA, Blalock TD, Balasubramanian L, Grotendorst GR, Schultz GS and Sherwood MB: Expression of connective tissue growth factor after glaucoma filtration surgery in a rabbit model. Invest Ophthalmol Visual Sci 45: 485-491, 2004.

This work is licensed under a Creative Commons Attribution-NonCommercial-NoDerivatives 4.0 International (CC BY-NC-ND 4.0) License. 